\title{
Tension Hemothorax following Blunt Abdominal Aortic Injury: CT Imaging
}

Seiji Morita*, Hiromichi Aoki, Haruna Hirakawa, Tomoatsu Tsuji, Takeshi Yamagiwa and Sadaki Inokuchi

Department of Emergency and Critical Care Medicine, Tokai University School of Medicine, 143 Shimokasuyalsehara-City Kanagawa, Japan

Blunt abdominal aortic injury is an extremely rare and fatal condition [1,2]. Hemothorax with bleeding from an extrathoracic organ is also an extremely rare condition [3]. Studies have reported abdominal aortic aneurysm and renal trauma but not hemothorax following blunt abdominal aortic injury.

We examined a 52-year-old woman with abdominal pain caused by seatbelt injury. On admission to our hospital, she was close to experiencing cardio-pulmonary arrest. Therefore, in the emergency department, immediately after intubation, we performed a lateral thoracotomy and clamped the descending thoracic aorta. Massive blood flow was observed from the left thoracic cavity; major bleeding occurred from a laceration of the posterior parietal pleura. We performed a right tube thoracostomy. At the same time, an intra-aortic balloon was inserted. A chest roentgenogram showed a right tension hemothorax (Figure 1a). After occlusion of the descending aorta, systolic blood pressure became $70-80 \mathrm{mmHg}$. We could not determine the location of the main source of bleeding. We decided to perform computed tomography (CT) and then perform an emergency operation on the CT table. Thoracic and abdominal CT scans are shown in Figure $1 \mathrm{~b}$ and $1 \mathrm{c}$. We performed emergency clamshell thoracotomy to clamp the aorta and achieve hemostasis. However, no thoracic organ injury

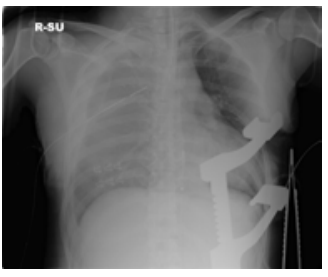

$1 \mathrm{a}$

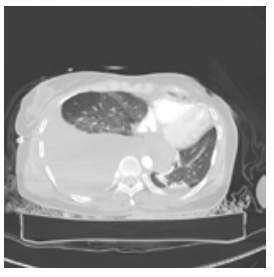

$1 \mathrm{~b}$

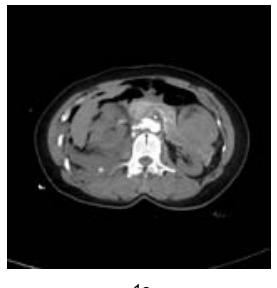

$1 \mathrm{c}$
Figure 1: was observed. Major bleeding occurred from a posterior parietal pleura laceration, which extended to the retroperitoneal space. Despite the emergency laparotomy, the patient could not be resuscitated.

We think that the abdominal aorta was pressed against the lumbar vertebrae by the seatbelt; a retroperitoneal hematoma from the ruptured abdominal aorta spread to the extrapleural space and ruptured the thoracic cavity, thereby causing tension hemothorax. In cases of massive hemothorax, it is necessary to consider the possibility of bleeding from an extrathoracic organ.

\section{References}

1. Borioni R, Garofalo M, Seddio F, Colagrande L, Marino B, et al. (1999) Posttraumatic infrarenal abdominal aortic pseudoaneurysm. Tex Heart Inst $J$ 26: 312-314.

2. Blanco FC, Powell DM, Guzzetta PC, Burd RS (2011) Aortic bifurcation rupture after blunt abdominal trauma in a child: a case report. J Pediatr Surg 46: 1452 1454.

3. Levy IG, Saadia R, Boffard KD (1988) Right-sided diaphragmatic rupture with herniation of the kidney presenting as an uncontrolled haemothorax--a case report. Injury 19: 293.
*Corresponding author: Seiji Morita, Department of Emergency and Critical Care Medicine, Tokai University School of Medicine, 143 Shimokasuyalsehara-City Kanagawa, Japan; Tel: 81463-93-1121; E-mail: morita@is.icc.u-tokai.ac.jp

Received December 02, 2012; Accepted June 12, 2012; Published June 15 2012

Citation: Morita S, Aoki H, Hirakawa H, Tsuji T, Yamagiwa T, et al. (2012) Tension Hemothorax following Blunt Abdominal Aortic Injury: CT Imaging. J Trauma Treat 1:138. doi:10.4172/2167-1222.1000138

Copyright: (c) 2012 Morita S. This is an open-access article distributed under the terms of the Creative Commons Attribution License, which permits unrestricted use, distribution, and reproduction in any medium, provided the original author and source are credited. 